\title{
Reglas de decisión para la selección de sistemas de citas basadas en características del paciente y de la institución de salud
}

\author{
JUAN PEDRO SEPÚLVEDA R. .,2,a, CRISTIÁN BERROETA M. ${ }^{\mathrm{b}}$
}

\section{Decision rules to schedule patient appointments}

Background: Outpatient scheduling has a significant impact on the perceived quality of service by the users and the efficient use of resources in the health system. There are mathematical methods that assist in solving this problem, but are seldom applied. Aim: To propose decision rules that are based on the own conditions of each institution and indicate which appointment system is the most suitable for the decision makers. Material and Methods: Through computer simulation, the effect of a wide range of decision and environmental factors over the appointment systems performance was assessed, in order to determine how these factors affect them. Results: Considering performance indicators associated to the patient's satisfaction and resources utilization, scheduling shorter length patients (e.g. check-up patients) in the beginning of the working day resulted to be in the efficient solutions frontier, as well as scheduling patients in one person blocks (shifting to multiple patient blocks only if resources utilization indicators are prioritized). Conclusions: Performance indicators are more sensitive to the sequence used to schedule different length patients, rather than the number of patients scheduled per block. Moreover, decision rules based on the institution priorities are proposed, which are quite robust to environmental factors.

(Rev Med Chile 2012; 140: 867-872).

Key words: Computer simulation; Outpatients; Point-of-care systems.

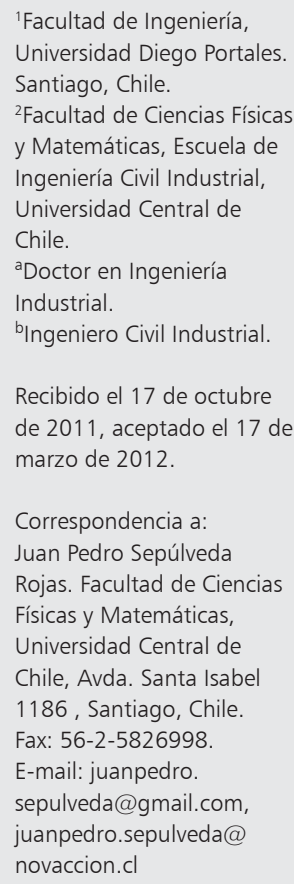

E ste trabajo aborda la problemática de programación de consultas de pacientes ambulatorios. En términos generales, una programación de citas consiste en determinar la hora para la cual se citará a cada paciente.

Hemos podido observar, principalmente en el ámbito público, que esta programación tiene un impacto significativo en la percepción de calidad de servicio por parte de los usuarios. Por ejemplo, las listas de espera para obtener una hora con ciertos especialistas, han obligado a largas esperas por parte de los usuarios. Dejando de lado otras variables, creemos que el eficiente uso de los recursos disponibles se vuelve un imperativo para poder entregar servicios de calidad para los usuarios del sistema de salud.

El objetivo es que esta programación rinda los mejores resultados (de acuerdo a las preferen- cias del tomador de decisión). Para resolver este problema, se han utilizado entre otros enfoques, optimizaciones matemáticas y heurísticas. Las primeras consisten en la construcción de un modelo matemático que luego se optimiza a través de algoritmos computacionales. La ventaja de estos métodos es que determinan la mejor solución (óptima) dentro de todas las posibles. Su desventaja es que la solución encontrada muchas veces difiere de la realidad, debido a que estos modelos suponen certitud completa de los datos y establecen una gran cantidad de supuestos. Por ejemplo, que todas las atenciones tengan la misma duración, que los pacientes llegan puntualmente y que no llegan pacientes espontáneos ${ }^{1,2}$. Por otro lado, las heurísticas consisten en algoritmos que no aseguran una solución óptima al problema, sin embargo, su ventaja está en su facilidad de apli- 
cación, la cercanía con el sistema real y el menor tiempo y costo que se necesita para encontrar una "buena" solución.

A pesar de la existencia de los métodos mencionados, en la práctica, estos métodos han sido escasamente aplicados. La aplicabilidad de estos métodos exige trabajo multidisciplinario, comenzando con un conocimiento profundo de la realidad entregado por los profesionales de la salud, luego, con esta información, el ingeniero procede a modelar el problema de la forma más fiel posible. Este modelo debe ser conciliado entre el médico y el ingeniero, para posteriormente analizar los resultados y obtener conclusiones de forma conjunta. Esta forma de trabajo, a nuestro juicio, puede garantizar el éxito en la aplicación de estas técnicas. Sin este trabajo en equipo, es probable que exista cierto nivel de incertidumbre en cuanto a los beneficios del uso de estas técnicas. Por ejemplo, algunas dudas que podrían plantearse son: ¿Los modelos propuestos en la literatura debieran tener mejores resultados en mi institución, considerando que fueron obtenidos bajo condiciones diferentes?; entre tantos modelos propuestos en la literatura, ¿cómo podría saber cuál es el mejor adaptado a las condiciones de mi institución? Un diagnóstico similar es presentado $\mathrm{en}^{3}$, quienes mencionan algunos factores que afectan el sistema de citas:

- Servicios entregados: Pre examinación, post examinación, realización de exámenes.

- Número de doctores.

- Número de citas por sesión.

- Proceso de llegada de pacientes: Impuntualidad, ausencias, llegada espontánea de pacientes, presencia de acompañantes.

- Tiempos de atención (en la consulta).

- Tardanza.

- Nivel de interrupción de los doctores.

- Prioridad en la atención.

Es por esto que se busca generar reglas de decisión para orientar la elección del mejor método de programación de citas, dadas las condiciones propias de cada institución de salud. Se intenta, además, conservar una jerga médica y presentar los resultados en forma práctica.

Nuestro trabajo sigue en la línea de estudios anteriores acerca de reglas de decisión para el diseño de sistemas de citas $^{4-5}$. Ho y $\mathrm{Lau}^{4}$ comparan 9 diferentes sistemas de citas en 27 diferentes escenarios clínicos caracterizados por 3 factores ambientales (probabilidad de ausencias, variación de tiempos de atención y número de pacientes por sesión). La medida de desempeño utilizada por estos autores fue la suma ponderada de tiempo ocioso de los médicos y el tiempo de espera de los pacientes. Los resultados de este estudio mostraron que un sistema de citas bien diseñado depende de las características de la institución y de la razón entre los costos del tiempo de espera de paciente y los costos del tiempo ocioso del médico. Los autores presentan los resultados en la forma de una frontera eficiente para los diferentes sistemas de citas utilizados.

Otros autores ${ }^{5}$ complementan el trabajo anterior $^{4}$ a través del análisis de un problema similar con menos restricciones, nuevos sistemas de citas y agregando otros indicadores de desempeño. Además incrementan los factores ambientales (agregando llegadas espontáneas y puntualidad de pacientes). También consideraron reglas de secuencia en el diseño del sistema de citas. Es decir, además de clasificar los pacientes, se les asigna durante la sesión de acuerdo a esta clasificación. Entonces, mientras que con la regla de cita se obtiene el tiempo entre citas, cuantos pacientes se atienden por bloque y el tamaño del bloque inicial, con las reglas de secuencia se puede definir el orden de asignación de cada paciente (ingreso o control, por ejemplo) dentro de la agenda. Con respecto a los indicadores de desempeño, estos autores $^{5}$ incluyen el sobretiempo del médico a los indicadores ya existentes (tiempo ocioso del médico y tiempo de espera de pacientes).

Otros autores ${ }^{6}$ estudian la variabilidad de tiempos de atención y el proceso de llegada de pacientes para estimar si un sistema abierto de citas (es decir, sólo existen llegadas espontáneas) es mejor que un sistema de citas tradicional. Un sistema de citas tradicional es más útil, según los autores, si el promedio de los tiempos de atención es relativamente grande y la impuntualidad es pequeña. En caso contrario, un sistema abierto de citas tiene un mejor desempeño. Con respecto a la variabilidad de los tiempos de atención (o tiempos de servicio), ellos mencionan que si ésta es pequeña, la utilidad de un sistema tradicional de citas es mayor.

En particular, en nuestro estudio, se extienden los análisis propuestos en la literatura considerando un conjunto superior de factores ambientales 
Reglas de decisión para citación de pacientes - J. P. Sepúlveda et al

e indicadores de desempeño, con el objetivo de desarrollar reglas de decisión más robustas para los tomadores de decisiones.

\section{Material y Métodos}

Para lograr el objetivo de este estudio (diseñar un sistema de citas), se investigó el efecto de las decisiones basadas en: las reglas de secuencia (en qué orden programar a los pacientes), las reglas de citas (número de pacientes citados por bloque de tiempo), y los factores ambientales. Se analizó un conjunto de valores de cada factor ambiental y se determinó cómo y en qué manera éstos afectan el desempeño de un sistema de citas específico.

Para ello se extendió el análisis realizado por otros autores ${ }^{5}$, incluyendo finalmente en nuestro análisis 5 factores ambientales: probabilidad de ausencia de pacientes ( $\mathrm{Pn}$ ), probabilidad de llegadas espontáneas $(\mathrm{Pw})$, puntualidad de pacientes (Punc), coeficiente de variación de tiempos de servicio $(\mathrm{CV})$ para pacientes nuevos (CVnew) y para pacientes de retorno o controles (CVret) y número de pacientes por sesión cada día (Num). Con respecto a las decisiones, se consideraron 3 reglas de citas y 3 reglas de secuencia, resultando un total de 9 sistemas de citas a analizar. La elección de estas reglas fue hecha basada en el buen desempeño de éstas mostrado en la literatura. Finalmente, los indicadores de desempeño utilizados fueron: tiempo de espera de pacientes, tiempo ocioso del médico, sobretiempo del médico y desviación estándar de los tiempos de espera de los pacientes.

Las reglas de secuencia escogidas fueron: ALTER, NWBG y RTBG. ALTER secuencia a pacientes nuevos $(\mathrm{N})$ y de retorno $(\mathrm{R})$ en orden alternado (RNRNRNR...); NWBG secuencia a los pacientes nuevos al comienzo de la sesión y los de retorno en el espacio remanente de la agenda (NNN... RRRR...); y RTBG secuencia a los pacientes de retorno al comienzo de la sesión (RRRR...NNN). En muchos sistemas de citas, los pacientes nuevos o ingresos utilizan más tiempo de atención que los de retorno. Es importante recalcar que muchos sistemas de citas pueden considerar una clasificación mayor a ésta, es decir, con más de dos diferentes tipos de pacientes. Sin embargo, para los objetivos del trabajo consideramos que no es necesario utilizar más de dos tipos, ya que la diferencia radica en los tiempos de atención entre un tipo de paciente y otro.

Con respecto a las reglas de cita se escogieron: IBFI, 2BEG y MBFI. La regla de cita IBFI (Individual-block/fixed-interval) agenda pacientes individualmente a intervalos iguales a el promedio del tiempo de atención del paciente. La regla $2 \mathrm{BEG}$ es una regla que cita a pacientes individualmente a intervalos fijos de tiempo, pero considerando un bloque al inicio de la sesión de 2 pacientes y la regla MBFI (multiple-block/fixed-interval) agenda 2 pacientes cada intervalo de tiempo, éste determinado por el promedio del tiempo de atención de los 2 pacientes.

Se utilizó simulación para testear la interacción entre los diferentes sistemas de citas y los factores ambientales. Los datos considerados para la simulación fueron obtenidos de la literatura ${ }^{3-5,7}$, basados principalmente en aquellos valores que, a nuestro juicio y de acuerdo a la opinión de médicos con experiencia en el área, podrían ser los más representativos para un amplio rango de instituciones de salud (Tabla 1).

Se realizaron 4.500 réplicas simuladas sobre un total de 576 escenarios ( 9 sistemas de citas por un total de 64 combinaciones diferentes de valores de los factores ambientales). Además una sesión diaria se consideró de una extensión de 3,5 horas (por ejemplo, una mañana o una tarde). El porcentaje de pacientes nuevos del total agendado fue

Tabla 1. Factores ambientales y sus correspondientes valores utilizados en la simulación

\begin{tabular}{|lcc|}
\hline Parametro & Valor $\mathbf{1}$ & Valor 2 \\
\hline Probabilidad de ausencia (\%) & 0 & 15 \\
Puntualidad (h) & Normal $(0 ; 0,41677)$ & Normal $(-0,25 ; 0,41677)$ \\
Coeficiente de variación de los tiempos de servicio (New y Ret), (\%) & 35 & 70 \\
Probabilidad de espontáneos (\%) & 0 & 15 \\
Número de pacientes por sesión (Num) & 10 & 20 \\
\hline
\end{tabular}


Tabla 2. Tiempos de atención del médico por tipo de paciente (horas)

\begin{tabular}{|lcccc|}
\hline Tipo de paciente & $\begin{array}{c}\text { Número de pacientes por sesión }=\mathbf{1 0} \\
\text { C.V. }=\mathbf{3 5} \%\end{array}$ & C.V. $=\mathbf{7 0} \%$ & \multicolumn{2}{c|}{ Número de pacientes por sesión $=\mathbf{2 0}$} \\
Nuevo & Lognormal & Lognormal & Lognormal & C.V. $=\mathbf{7 0} \%$ \\
& $(0,5 ; 0,175)$ & $(0,5 ; 0,35)$ & $(0,25 ; 0,0875)$ & Lognormal \\
Retorno & Lognormal & Lognormal & Lognormal & $(0,25 ; 0,175)$ \\
& $(0,25 ; 0,0875)$ & $(0,25 ; 0,175)$ & $(0,125 ; 0,04375)$ & $(0,125 ; 0,0875)$ \\
Espontáneo & Lognormal & Lognormal & Lognormal & Lognormal \\
& $(0,35 ; 0,1225)$ & $(0,35 ; 0,245)$ & $(0,175 ; 0,06125)$ & $(0,175 ; 0,1225)$ \\
\hline
\end{tabular}

Obs: Una sesión tiene una duración fija de 3,5 horas cronológicas.

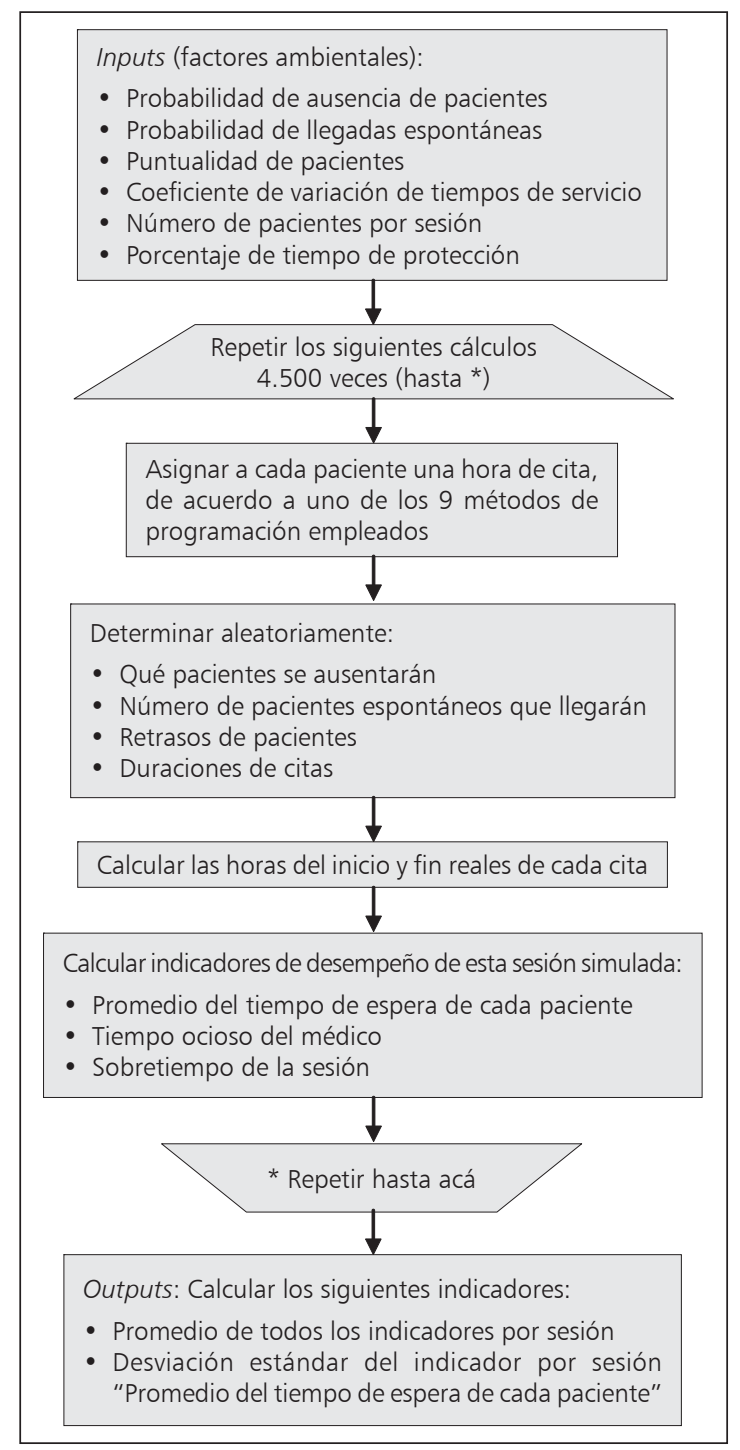

Figura 1. Representación General del Funcionamiento del Modelo de Simulación Elaborado. fijado en $40 \%$. Finalmente, los tiempos de atención del médico fueron fijados de acuerdo a la Tabla 2.

Finalmente, la Figura 1 explica, en términos generales, el modelo de simulación elaborado, el cual se ejecutó para cada método de programación testeado.

\section{Resultados}

La Tabla 3 muestra el porcentaje de escenarios en el cual la combinación regla de cita-regla de secuencia es parte de la frontera de soluciones eficientes (las combinaciones que se encuentran en la frontera de soluciones eficientes son aquéllas que son mejores que cualquier otra combinación).

Por ejemplo, en $91 \%$ de las 192 combinaciones de factores ambientales y decisionales en las cuales la secuencia RTBG fue utilizada, ésta estuvo en la frontera eficiente. RTBG entonces tiene mucha mayor presencia que las otras reglas de secuencia en la frontera de soluciones eficientes. Con respecto a las reglas de cita, la diferencia entre las 3 reglas no es tan neta, observándose una mayor presencia en la frontera de soluciones eficientes de 2BEG y MBFI.

En la Tabla 4 se presentan los valores promedio, sobre todos los escenarios, de los indicadores de desempeño para cada una de las 9 combinaciones regla de cita-regla de secuencia.

Se observa que en promedio, la mejor combinación para el tiempo de espera promedio del paciente fue RTBG-IBFI. Con respecto a la desviación estándar de los tiempos de espera de pacientes la mejor combinación de regla de cita y regla de secuencia fue RTBG-2BEG. Mientras que para los indicadores de sobretiempo y tiempo ocioso del médico, la mejor combinación fue RTBG-MBFI. 
Reglas de decisión para citación de pacientes - J. P. Sepúlveda et al

Tabla 3. Porcentaje de presencia en la frontera de soluciones eficientes de las reglas de cita y secuencia

\begin{tabular}{|lrrcc|}
\hline $\begin{array}{l}\text { Regla de } \\
\text { secuencia }\end{array}$ & 2BEG & IBFI & MBFI & Total \\
\hline ALTER & $3 \%$ & $0 \%$ & $6 \%$ & $3 \%$ \\
NWBG & $13 \%$ & $2 \%$ & $0 \%$ & $5 \%$ \\
\hline RTBG & $97 \%$ & $77 \%$ & $98 \%$ & $91 \%$ \\
\hline Total & $38 \%$ & $26 \%$ & $35 \%$ & \\
\hline
\end{tabular}

Tabla 4. Valores promedio ( $\mathrm{min}$ ) de indicadores

de desempeño para cada combinación regla de cita-regla de secuencia

\begin{tabular}{|lcccc|}
\hline $\begin{array}{l}\text { Regla de } \\
\text { secuencia; } \\
\text { regla de cita }\end{array}$ & $\begin{array}{c}\text { Tiempo de } \\
\text { espera del } \\
\text { paciente }\end{array}$ & $\begin{array}{c}\text { Sobre- } \\
\text { tiempo }\end{array}$ & $\begin{array}{c}\text { Tiempo } \\
\text { ocioso }\end{array}$ & $\begin{array}{c}\text { Desv. estándar del } \\
\text { tiempo } \\
\text { de espera }\end{array}$ \\
\hline Alter; $2 \mathrm{BEG}$ & 10,7 & 2,4 & 2,6 & 10,3 \\
\hline Alter; IBFI & 10,9 & 2,5 & 2,7 & 10,7 \\
\hline Alter; MBFI & 11,6 & 2,1 & 2,3 & 10,9 \\
\hline NWBG; $2 \mathrm{BEG}$ & 11,8 & 2,4 & 2,6 & 12,2 \\
\hline NWBG; IBFI & 12,0 & 2,5 & 2,8 & 12,9 \\
\hline NWBG; MBFI & 12,8 & 2,1 & 2,2 & 12,9 \\
\hline RTBG; $2 \mathrm{BEG}$ & 8,5 & 2,3 & 2,3 & $7,1^{*}$ \\
\hline RTBG; IBFI & $8,4^{*}$ & 2,4 & 2,4 & 7,3 \\
\hline RTBG; $M B F I$ & 10,4 & $1,9^{*}$ & $1,8^{*}$ & 8,0 \\
\hline
\end{tabular}

*Corresponde al mejor valor obtenido.

Se analizó además el impacto de los factores ambientales sobre la robustez de los resultados. Encontrándose que para todos los factores ambientales (exceptuando la puntualidad del paciente), independiente de los valores considerados, la mejor combinación de regla y secuencia fue lo suficientemente robusta como para no cambiarla.

\section{Discusión}

El objetivo de esta investigación fue la proposición de reglas de decisión (reglas de citas y reglas de secuencia de pacientes) que, basadas en condiciones específicas de cada institución de salud, indicara qué sistema de citas era más deseable en relación a un indicador de desempeño escogido por el tomador de decisiones. En este trabajo se extendió el análisis propuesto en la literatura considerando variables adicionales y más indicadores de desempeño, con el objetivo de desarrollar reglas de decisión más confiables para los tomadores de decisiones de las instituciones de salud.

Con respecto a las regla de secuencia, y de acuerdo a los valores de los factores ambientales utilizados en la simulación, se obtuvo que la secuencia RTBG (para cualquier combinación de reglas de citas) tiene desempeños que, en general, son muy superiores para todas las medidas de desempeño. Para los indicadores de desempeño relacionados con el paciente (promedio y desviación estándar del tiempo de espera del paciente) las combinaciones RTBG-IBFI y RTBG-2BEG, respectivamente, tuvieron un mejor desempeño. Esto 
significa que secuenciar pacientes de retorno al comienzo de la sesión con un bloque de 1 paciente en cada cita comenzando la sesión con un bloque inicial de 2 pacientes (2BEG) y 1 un paciente (IBFI) tienen un mejor desempeño cuando el tomador de decisiones desea enfatizar los indicadores relacionados con la satisfacción del paciente. Por el contrario, si el tomador de decisiones desea tener un mejor desempeño en indicadores relacionados con el uso de recursos (sobretiempo y tiempo ocioso del médico), la combinación RTBG-MBFI, es decir, agendar pacientes de retorno al comienzo de la jornada con un bloque de 2 pacientes por cada cita durante toda la sesión, tiene un mejor desempeño.

Además, se observa que los indicadores de desempeño, de acuerdo a nuestros resultados, son más sensibles a los factores de decisión (reglas de citas y secuencias) que a los factores ambientales (puntualidad, presencia de espontáneos, variabilidad de tiempos de servicio, etc.). Aquí se presenta una diferencia con algunos resultados de la literatura ${ }^{3-5}$, debido a que éstos consideran de igual importancia los factores ambientales y los factores de decisión.

Entonces, para casi todos los escenarios estudiados, la regla de secuencia con mejor desempeño fue la misma (RTBG). Para las reglas de cita, se encontró que múltiples pacientes por bloque (MBFI) tiene siempre un mejor de-sempeño para los indicadores de sobretiempo y tiempo ocioso. Este resultado es intuitivo, porque con múltiples pacientes por bloque horario, la probabilidad de que el médico esté en espera de un paciente es más baja. Para los indicadores relacionados con la satisfacción del paciente, las reglas de cita IBFI y 2BEG tuvieron mejor desempeño, debido a que, en promedio, el paciente deberá esperar menos tiempo que con respecto al caso de múltiples pacientes por bloque.

Así, de acuerdo a los resultados de la simulación, la regla de decisión para los tomadores de decisiones de instituciones de salud es: si el tomador de decisiones está focalizado en mejorar indicadores de desempeño relacionados con la satisfacción del paciente, deberá elegir entre los sistemas de citas RTBG-2BEG y RTBG-IBFI; y si éste está focalizado en indicadores de desempeño relacionados con la utilización de recursos (para este caso, el sobretiempo y tiempo ocioso del médico), deberá elegir el sistema de citas RTBGMBFI.

Estos resultados, a nuestro juicio, son generalizables a cualquier institución de salud, debido principalmente a la cantidad de factores ambientales, y variables de decisión consideradas. Sin embargo, y como perspectiva de continuación de este trabajo, estimamos necesario continuar este estudio realizando un análisis de sensibilidad de los resultados obtenidos para un amplio rango de valores de factores ambientales.

Agradecimientos. Quisiéramos agradecer al Doctor Sr. Ernesto Behnke, Director del Hospital Padre Hurtado, por sus valiosos comentarios en relación a este artículo.

\section{Referencias}

1. Kaandorp G, Koole G. Optimal outpatient appointment scheduling. Health Care Management Science 2007; 10 (3): 217-29.

2. Muthuraman K, Lawley M. A Stochastic Overbooking Policy for Open Access Clinical Scheduling. IIE Transactions 2008; 40 (9): 820-37.

3. Cayirli T, Veral E. Outpatient Scheduling in Health Care: A Review of Literature. Production and Operations Management 2003; 12 (4): 519-49.

4. Ho C-J, Lau H-S. Minimizing Total Cost in Scheduling Outpatient Appointments. Management Science 1992; 38 (12): 1750-64.

5. Cayirli T, Veral E, Rosen H. Designing appointment scheduling systems for ambulatory care services. Health Care Manage Science 2006; 9: 47-58.

6. Mondschein S, Weintraub G. ¿Bajo qué Escenarios es Conveniente Realizar Citaciones en una Empresa de Servicios? Revista Ingeniería de Sistemas 2002; 16 (1): 21-48.

7. Gupta D, Denton B. Appointment Scheduling in Health Care: Challenges and Opportunities. IIE Transactions 2008; 40: 800-19. 